\title{
Feature Level Fusion the Performance of Multimodal Biometric Systems
}

\author{
Harbi AlMahafzah \\ Department of Computer Science, \\ Al-Hussein Bin Talal University, \\ Ma'an, Jordan
}

\author{
Ma'en Zaid AlRawashdeh \\ Computer Lecturer,Alghad International Colleges \\ for Health Science, \\ Najran, Saudi Arabia
}

\begin{abstract}
This paper proposed the use of multimodal feature-level fusion to prove the improvement performance of multimodal authentication. Different algorithm used for features extraction, LG for extracting FKP features, LPQ for iris and Palmprint features extraction, and PCA for extracting face features. Results brought to light that the multimodal authentication process gained higher performance than single modality. The biometric performance using feature-level fusions under "Z-score", "Tanh", "Median", and Min-Max normalization has been demonstrated in this paper.
\end{abstract}

\section{General Terms}

Biometric

\section{Keywords}

Feature Level Fusion; Multibiometric; Multimodal; ; LPQ; PCA; Log-Gabor.

\section{INTRODUCTION}

As the concerns about security and vast progression in networking the need for user authentication techniques has increased in the back few decade. Traditional authentication methods are commonly used for authorizing and bind access to a different systems. These systems could be attacked and the security can be override. Biometrics technologies have replaced the traditional authentication methods as their ability to authenticated the right identity of different people requesting a service [13].

Biometric recognition systems aim to automation the recognition of a person's identity based on physical or behavioral characteristics (something a person is or produces).

Since majority of biometric systems are single in modality which rely on the single biometric information of authentication, which raise a problems with most of biometrics trait such as (noise in sensed data, intra-class variations, and inter-class similarities, etc.), as a result of the mentioned problems the recognizing unauthorized user as authorized users (FAR) and rejection authorized users (FRR). FAR\&FRR Usually used to measure the performance of biometric systems, another measurement which is (EER) is also could be used, EER is cross point when drawing FRR verses FAR(i.e. the equal values of FRR with FAR [3] .

Now a days there is more concern of fixing some inherited problems of a biometric systems (intra-class variations, interclass similarity etc.). A possible solution is to use more than one modality to reduce the classification problem which rise the intra-class variety and inter-class. Interlace or fusing more than one biometric refer as Multi-biometric [8].The Multibiometric systems can offer staple improvement in the authentication accuracy of a biometric system, as it rely on more than one biometric data.

Multi-biometrics refer as the fusion of different types of biometrics according to the way of fusing the biometrics data as follows[3]: Multi-sensor: Multiple sensors are used to collect information of the same biometric. Multi-sample: more than foresight, of the same biometric are taken at the time of the enrolment and/or recognizing time, e.g. a number of face readings are taken from different sides for the same person. Multi-algorithms: different algorithms used for extracting the same biometric trait features and matching with already achieved database. Multi-instance: means the use of the same biometric trait and processing on multiple instances of the analogous biometric trait, (such as left and right irises) $[1,13]$. Multi-modal: Multiple biometric modalities can be collected from the same person, e.g. fingerprint and face, which requires different sensors and may requires different algorithms.

Thus this paper evaluates the performance of multi-modal approach by fusing the data at feature score level using " $\mathrm{Z}$ score", "Tanh", "Median", and Min-Max normalization. The rest of the paper is organized as follows: Section 2 presents related works, proposed method is given in section 3, detailed experimental results are given in section 4 , fusion strategies in section 5 , result and discussion in section 6 , and conclusion is mentioned in section 7 .

\section{RELATED WORKS}

Zhifang Wang et al. [4]: have proposed a method to combine the face and iris features for promoting a biometric multimode system. The authors espouse an efficient feature level fusion scheme for fusing iris and face features in string, and normalizing the original features using $\mathrm{z}$-score to eliminate the unbalance of magnitude and the rating between two different kinds of feature vectors.

Mohamed K. Shahin et al. [5]: have introduced a multimodal biometric system based on fusion of entire dorsal geometry of hand and fingerprints that achieves right and left near-infrared dorsal geometry and right and left index and ring fingerprints. Scores acquired from different biometric matchers were fused using the Min-Max score fusion technique.

Nupriya Goyal et al. [12]: in their paper they presented and analyzed the prime works in multimodal biometric system and its capability in recognition rate. Also they proposed framework of the multimodal biometric system involve face and ear.

S.K.Bhardwaj [10]: in their paper the authors summarized the possibility to promote the person identification system by consolidate multiple biometric traits at feature level. They shows an algorithm for fusing a face and hand at feature level. 
The min-max normalization technique were used to normalize the features gained from face and hand.

Zhifang Wang et al. [9]: in their paper, proposed a feature level fusion algorithm of face and palm. The canonical correlation analysis (CCA) idea is used to extract the correlation feature of face and palm. The result showed that the algorithm ameliorate the recognition rate.

\section{PROPOSED METHODOLGY}

In this paper, different modalities have been used namely: Face modality of AR-Face database, iris modality of CASIAIris database, palmprint modality of PolyU-Palmprint (Pp) and Finger Knuckle Print (FKP) modality of DZhang FKP database. FKP refers to the image pattern of the outer surface around the phalangeal joint of one's finger..

\section{FEATURE EXTRACTION}

In this paper the subsequent feature extraction algorithms used to extract the features prior to fuse a different modalities sets.

- Log-Gabor filters used to extract finger knuckle print features. Log-Gabor function proposed by Field [6], Field suggests that natural images are best establish by filters that have Gaussian transfer functions on the logarithmic frequency scale. The log-Gabor on the linear frequency scale has a transfer function of the form:

$$
G(w)=e^{\left(-\log \left(w / w_{0}\right)^{2}\right) /\left(2\left(\log \left(k / w_{0}\right)^{2}\right)\right.}
$$

Where $w_{0}$ is the filter's center frequency. To gain constant shape ratio filters the term $k / w_{0}$ must also be held constant for varying $w_{0}$.

- Local phase quantization (LPQ) method used to extract the iris's and Palmprint's features. LPQ introduced by Ojansivu et al. [7]. LPQ is based on the blur invariance property of the Fourier phase spectrum. The image $f(x)$ defined by:

$$
F(u, x)=\sum_{y \in N_{x}} f(x-y) e^{-j 2 \pi u^{T} y}=w_{u}^{T} f_{x}
$$

Where $w_{u}$ is the basis vector of the 2-D DFT at frequency $u$, and $f_{x}$ is another vector containing all $M^{2}$ image samples from $N_{x}[7]$

- Principal component analysis (PCA) used to extract the face features. PCA was invented in 1901 by Karl Pearson.

\section{BIOMETRIC FUSION STATEGIES}

Biometric system works in two modes: enrollment and authentication. Verification and identification are the two modes an authentication can be accomplished. Fusion of biometric systems, algorithms and/or traits is a well-known solution to ameliorate authentication performance of biometric systems. Researchers have shown that multibiometrics, i.e., fusion of multiple biometric evidences, enhances the recognition performance.

In biometric systems; fusion can be carry out at different levels; Sensor Level, Feature Level, Score Level, and Decision Level Fusion [11].

\subsection{Sensor Level Fusion}

It presuppose the unification of evidence presented by multiple sources of raw data before they are submissive to feature extraction. Sensor level fusion can avail multi-sample systems which capture multiple snapshots of the same biometric trait.

\subsection{Feature Level Fusion}

In feature-level fusion, the feature sets generating from multiple biometric algorithms are conjoined into a single feature set by the implementation of appropriate feature normalization, transformation, and reduction schemes $[8,11]$. Feature level fusion is shown in Figure-3.

\subsection{Score Level Fusion}

The match scores generated by multiple biometric matchers are consolidated to generate a new match score (a scalar).

\subsection{Decision Level Fusion}

Fusion is carried out at the abstract or decision level when final decisions are obtainable (e.g. AND, OR, Majority Voting, etc.).

In all the experiments, the data have been fused at feature level, using using "Z-score", "Tanh", "Median", and MinMax normalization.

\section{RESULT AND DISCUSSION}

This section deals with the fulfillment outcome of combining different biometrics modalities at feature level fusion with "Z-score", "Tanh", "Median", and Min-Max normalization to mensuration the performance of multimodal system.

Z-Score; $\quad n s_{j}^{t}=\frac{s_{j}^{t}-\mu_{j}}{\sigma_{j}}$

Tanh-Score; $n s_{j}^{t}=1 / 2\left\{\tanh \left(0.01\left(\frac{s_{j}^{t}-\mu_{j}}{\sigma_{j}}\right)\right)+1\right\}$

Median-Score; $\quad n s_{j}^{t}=\frac{s_{j}^{t}-m e d_{j}}{M A D_{j}}$

Where: $\operatorname{med}_{j}=\operatorname{median}_{i=1}^{N} s_{j}^{t}$, and

$$
M A D=\operatorname{median}_{i=1}^{N}\left|s_{j}^{t}-\operatorname{med}_{j}\right|
$$

Min-Max Score; $n s_{j}^{t}=\frac{s_{j}^{t}-\min _{i=1}^{N} s_{j}^{t}}{\max _{i=1}^{N} s_{j}^{t}-\min _{i=1}^{N} s_{j}^{t}}$

In all the experiments, performance were measure by False Acceptance Rate (FAR in \%) and corresponding Genuine Acceptance Rate (GAR in \%). Firstly the performance of a single modality biometric system is measured; subsequent the results for multimodal biometric system are estimated. The results acquired from single modality biometric system are tabulated in Table1, and depicted as Receiver Operating Characteristic (ROC) curve in Figure.2.

Table 1. Performance of Single Modality

\begin{tabular}{ccccc}
\hline \multicolumn{5}{c}{ GAR (\%) } \\
\hline FAR (\%) & FKP & PalmPrint & Iris & Face \\
\hline 0.01 & 85.50 & 86.00 & 45.00 & 25.50 \\
\hline 0.10 & 88.50 & 88.50 & 57.00 & 40.00 \\
\hline 1.00 & 93.00 & 97.00 & 74.00 & 32.00 \\
\hline
\end{tabular}

From above table (table-1) which list the single modality data it can be seen that the palmprint and FKP have the highest performance out of the four modalities for all the FAR values. But Palmprint outperforms the FKP at $\mathrm{FAR}=1$. Performance of iris is accepted at $F A R=1$ but has low value at $F A R=0.01$ compare to FKP and palmprint. Whereas the face has a less performance values than other modalities at all values of 
FAR.

The results acquired from two modality biometric systems are tabulated in Table-2, and presented graphically as Receiver Operating Characteristic (ROC) curve in Figure-3.

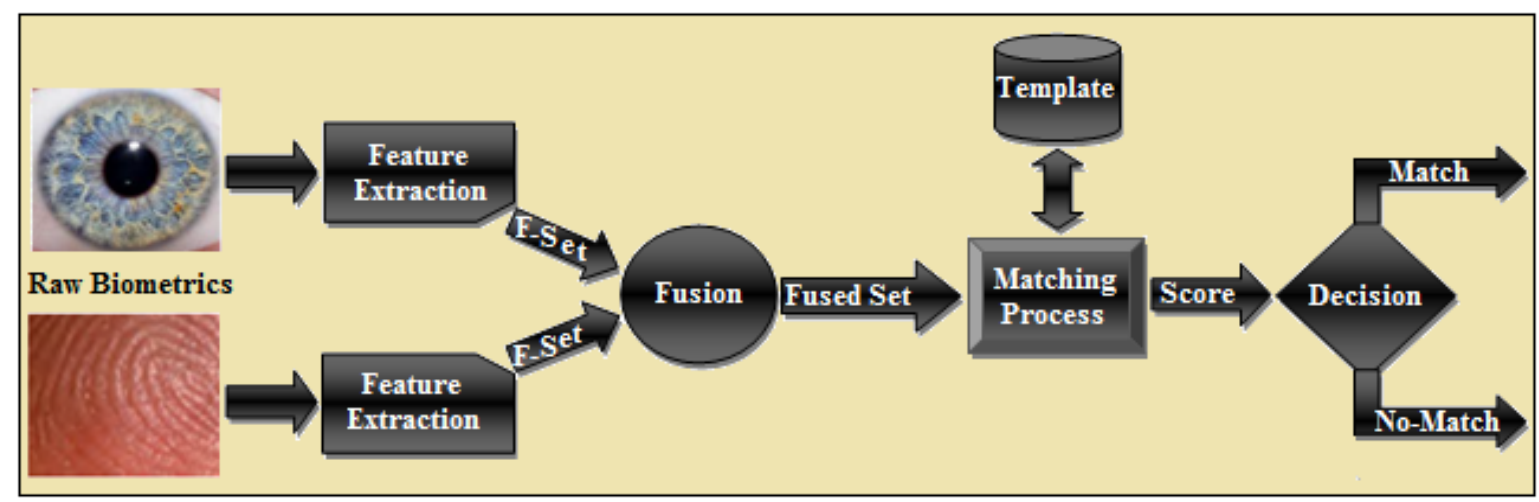

Fig. 1. Basic Concept of the Feature Level Fusion

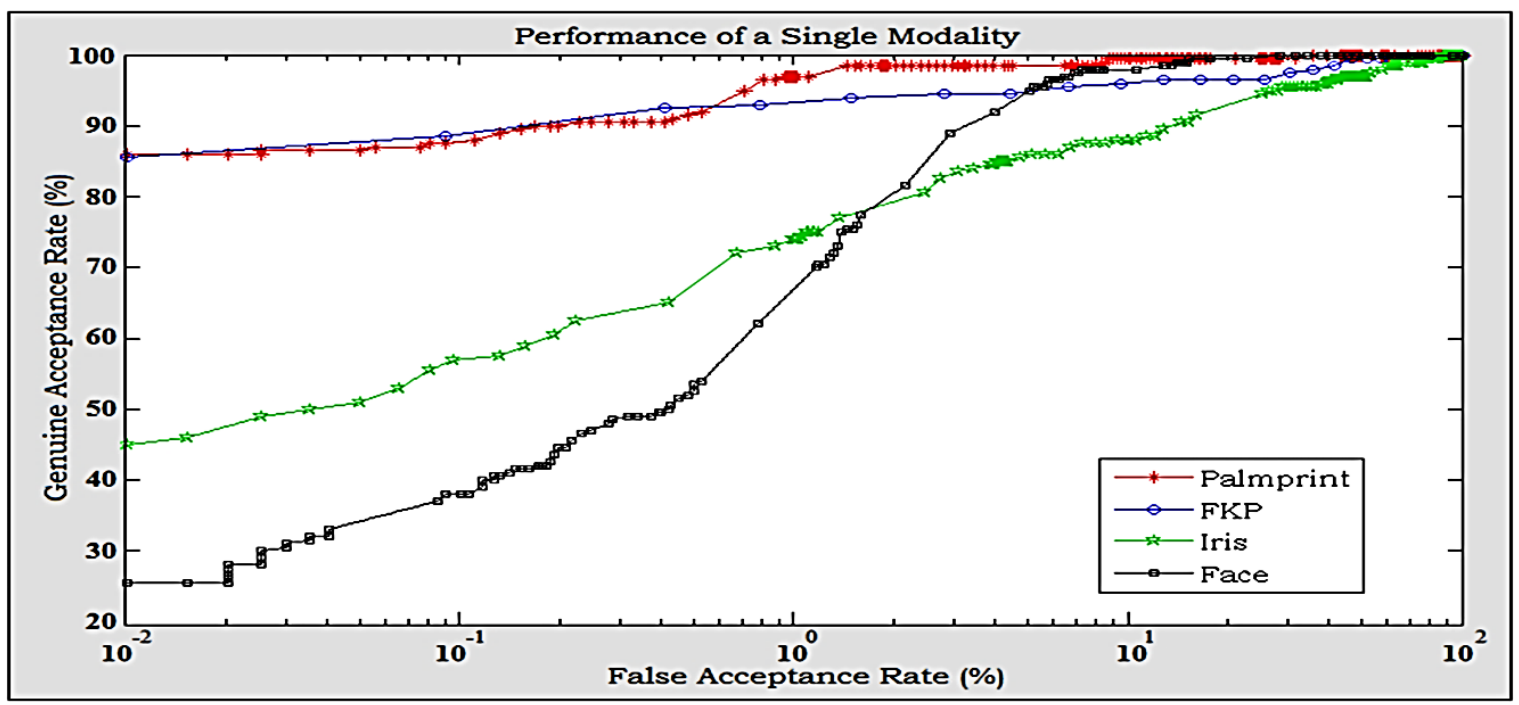

Fig. 2. The ROC Curve Performance of Single Modality

Table 2. Performance of Two Modality Biometric Systems

\begin{tabular}{|c|c|c|c|c|c|c|}
\hline \multirow{2}{*}{ FAR $(\%)$} & \multicolumn{6}{|c|}{ GAR (\%) With "Z-score" Normalization } \\
\hline & FKP+Palm & FKP+Face & FKP+Iris & Palm+Iris & Palm+Face & Iris+Face \\
\hline 0.01 & 86.50 & 18.00 & 86.50 & 10.00 & 18.00 & 18.00 \\
\hline $\mathbf{0 . 1 0}$ & 89.50 & 34.00 & $\mathbf{8 9 . 5 0}$ & 12.50 & 34.00 & 34.00 \\
\hline 1.00 & 92.00 & 75.50 & 92.00 & 48.00 & 75.50 & 75.50 \\
\hline \multicolumn{7}{|c|}{ GAR (\%) With "Tanh-estimator" Normalization } \\
\hline 0.01 & 86.50 & 18.00 & 85.50 & 10.00 & 18.00 & 18.00 \\
\hline $\mathbf{0 . 1 0}$ & 89.50 & 34.00 & $\mathbf{8 8 . 5 0}$ & 12.50 & 34.00 & 34.00 \\
\hline 1.00 & 92.00 & 75.50 & 91.00 & 48.00 & 75.50 & 75.50 \\
\hline \multicolumn{7}{|c|}{$\begin{array}{c}\text { GAR (\%) With "Median and } \\
\text { Median Absolute Normalization" Normalization }\end{array}$} \\
\hline 0.01 & $\mathbf{8 5 . 0 0}$ & 25.00 & 84.00 & 65.00 & 26.00 & 25.00 \\
\hline 0.10 & 87.00 & 40.00 & 86.00 & 77.00 & 38.50 & 39.00 \\
\hline 1.00 & 92.50 & 60.50 & 91.50 & 87.00 & 63.50 & 71.50 \\
\hline \multicolumn{7}{|c|}{ GAR (\%) With "Min-Max-score" Normalization } \\
\hline 0.01 & 71.00 & 03.00 & 71.00 & 09.00 & $\mathbf{0 7 . 0 0}$ & 07.00 \\
\hline 0.10 & 78.00 & 10.00 & 78.00 & 09.00 & 15.00 & 15.00 \\
\hline 1.00 & 86.50 & 23.00 & 86.50 & 33.00 & 37.00 & 37.00 \\
\hline
\end{tabular}


From Table-2 it can be observed that the fusion of two modality does not have any significant score improvement for a good performance modality (FKP/Palmprint) when fused together under either Z-score or tanh-estimators, and has low performance when combining a good performance modality with low performance one except for FKP\&Iris which has the same values as the FKP itself. But does not have improvement with Median \& MAD which is less than the highest performance of the fused modality except for fusing
Palm\&Iris compared to Iris as a single but still is less than the values of Palm as single modality. With Min-Max normalization the performance retreating down. Figure-3 shows the ROC curve for the performance fusion of two algorithms at Feature level with different normalization techniques

The results obtained from three modality biometric systems are tabulated in Table-3.
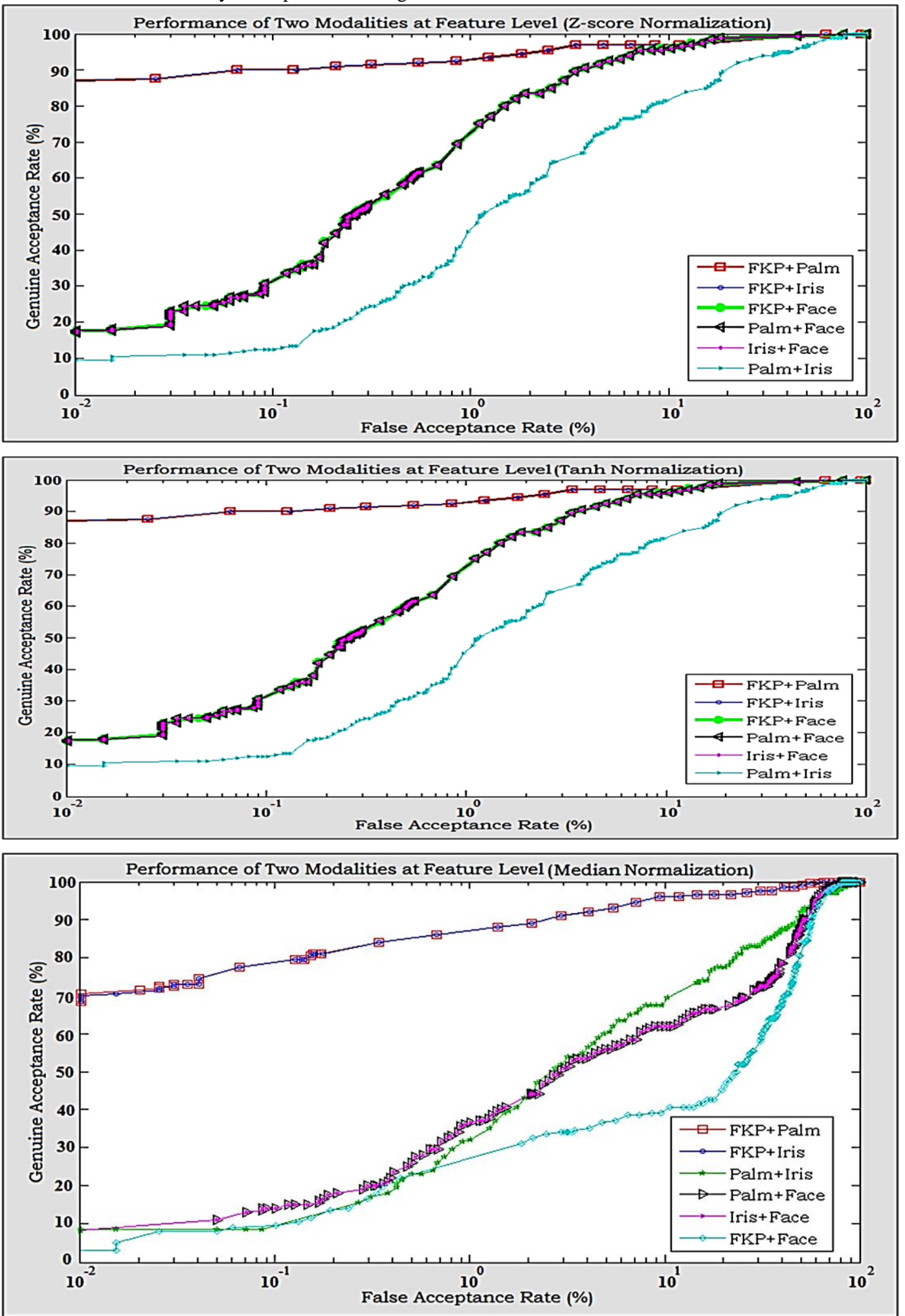


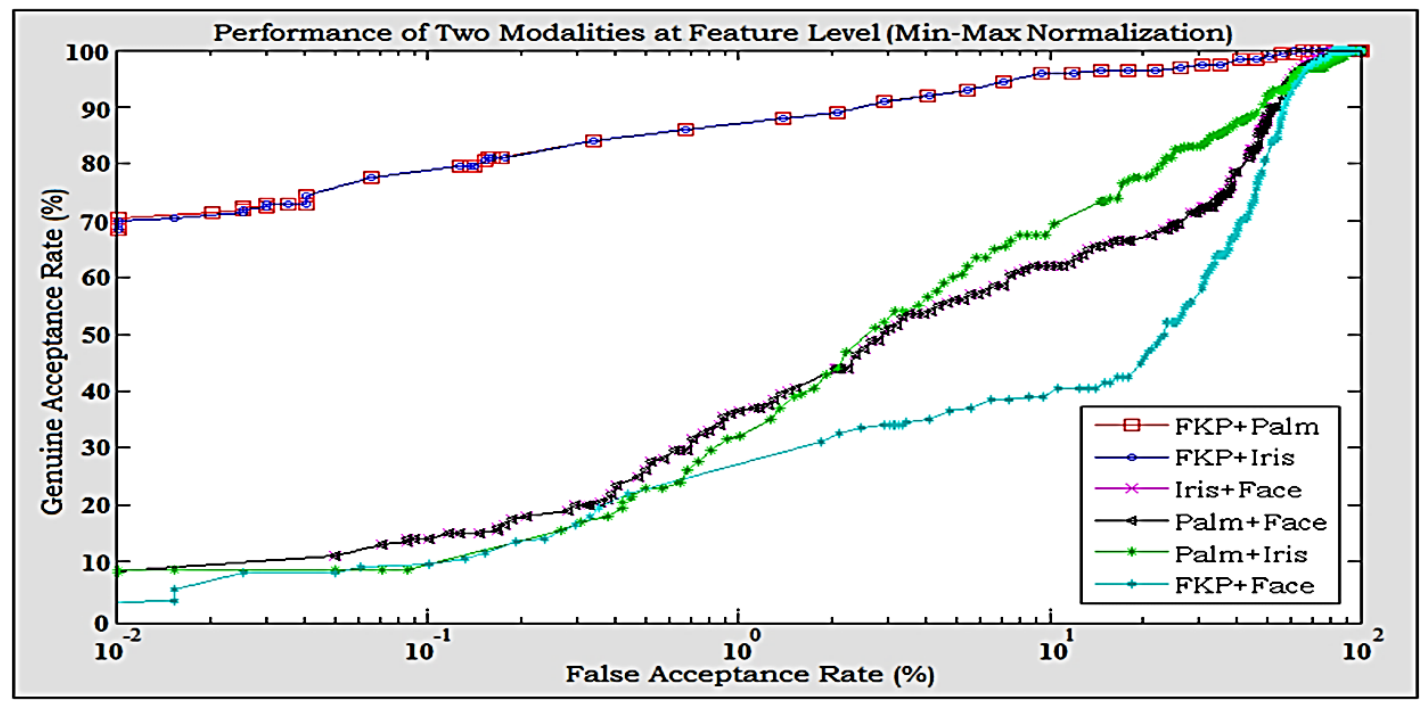

Fig. 3. The ROC Curve Performance of Two Modality Systems at Feature Level

Table 3. Performance of Three Modality Biometric Systems

\begin{tabular}{|c|c|c|c|c|}
\hline \multirow{2}{*}{$\operatorname{FAR}(\%)$} & \multicolumn{4}{|c|}{ GAR (\%) With "Z-score" Normalization } \\
\hline & FKP+Palm+Iris & FKP+Palm+Face & FKP+Iris+Face & Palm+Iris+Face \\
\hline 0.01 & 87.00 & 18.00 & $\mathbf{1 7 . 0 0}$ & 16.00 \\
\hline $\mathbf{0 . 1 0}$ & 90.00 & 35.00 & 34.00 & 33.00 \\
\hline 1.00 & 93.00 & 76.00 & 75.00 & 74.00 \\
\hline \multicolumn{5}{|c|}{ GAR (\%) With "Tanh-estimator" Normalization } \\
\hline 0.01 & 88.00 & 19.00 & 18.00 & 16.50 \\
\hline $\mathbf{0 . 1 0}$ & 91.00 & 35.50 & 35.00 & 34.00 \\
\hline 1.00 & 93.50 & 77.00 & 75.50 & 75.00 \\
\hline \multicolumn{5}{|c|}{$\begin{array}{l}\text { GAR (\%) With "Median and } \\
\text { Absolute Normalization" Normalization }\end{array}$} \\
\hline 0.01 & 86.00 & 25.00 & 24.00 & 25.50 \\
\hline 0.10 & 89.00 & 40.00 & 39.00 & 41.00 \\
\hline 1.00 & 94.00 & 69.00 & 68.50 & 70.00 \\
\hline \multicolumn{5}{|c|}{ GAR (\%) With "Min-Max-score" Normalization } \\
\hline 0.01 & 71.00 & 03.50 & 03.00 & 10.00 \\
\hline $\mathbf{0 . 1 0}$ & 78.00 & $\mathbf{1 0 . 0 0}$ & $\mathbf{1 0 . 0 0}$ & 15.00 \\
\hline 1.00 & 86.00 & 22.00 & 21.50 & 33.00 \\
\hline
\end{tabular}

From the above table (table-3) it can be seen that for all for all the normalization technique the fusion of three modality except of combining the two good performed modality with moderated one (FKP+Palm+Iris) which has the same performance as a single one. For the rest of combination is has significant fall down of a score. The results obtained from three modality biometric systems depicted as Receiver Operating Characteristic (ROC) curve in Figure-4.

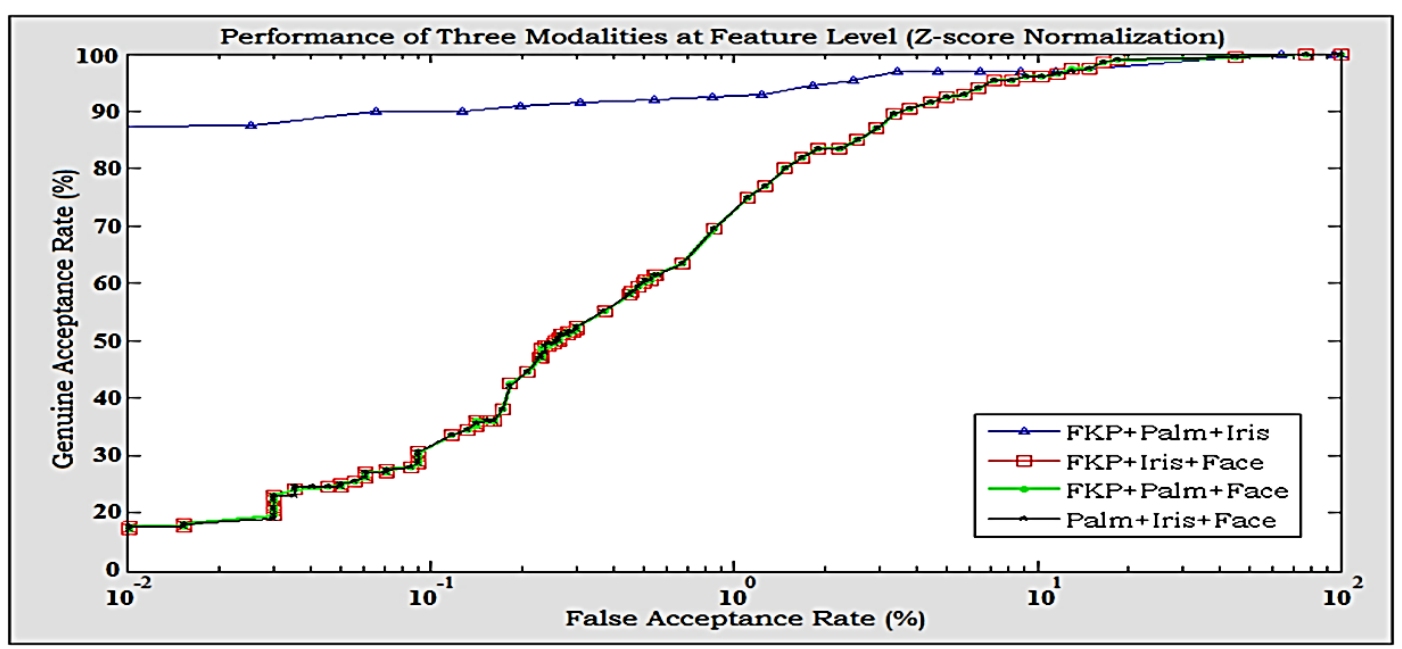



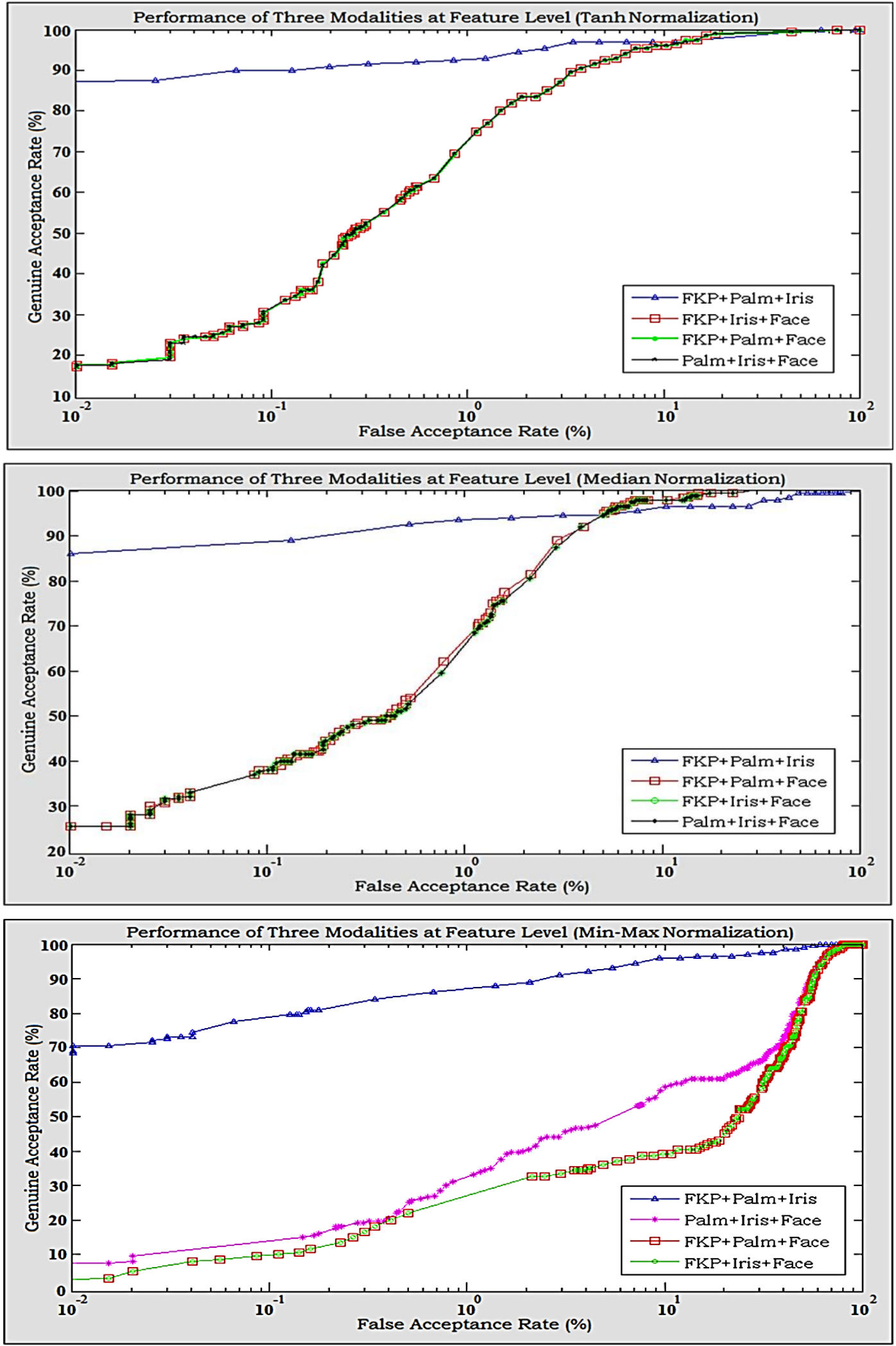

Fig. 4. The ROC Curve Performance of Three Modality Systems at Feature Level

\section{CONCLUSION}

Based on the analysis of experimental results and observations, it can be concluded that the performance fusion of two modalities at feature level "Z-score and Tanh" has almost the same score as a single modality at the best case. While the performance of the most combinations has gone down. The three combinations of all the modality has gone down for the normalization technique. As we have seen in our previous papers the performance of multimodality at score and decision levels of fusion has a good performance compare to single biometric trait.

We could conclude that the feature level is degrading the 
performance fusion of modalities, it could be due to the different features which combined together and according to that the decision is taken.

Since the design of a multibiometric system depends on several factors, including choices of modality, feature extraction algorithms, the modalities to be combined, and the fusion strategy to be applied. Generally, it is hard to prognosticate the exemplary biometric trait pertinent to a particular application, even it is hard to mensuration the choice of feature extraction algorithm and adequate fusion methodology based on recognition performance alone. Thus in the future work we are going to examine more modality and different algorithms for each one to be able to select the best algorithm for each modality and choose the best combination between the modalities hence we can try to reach a $100 \%$ matching based on the way of fusing a different modalities.

\section{REFERENCES}

[1] Stan, J., Li Z., Jain, A.K. "Encyclopedia of Biometrics". Springer.

[2] Tiago Sanches, João Antunes, Paulo Lobato Correia"A Single Sensor Hand Biometric Multimodal System". 15th European Signal Processing Conference (EUSIPCO 2007), Poznan, Poland, September 3-7, 2007, copyright by EURASIP.

[3] Andrew Teoh, S. A. Samad and A. Hussain, "Nearest Neighbourhood Classifiers in a Bimodal Biometric Verification System Fusion Decision", Journal of Research and Practice in Information Technology, Vol. 36, No. 1, February 2004.

[4] Zhifang Wang, Jie Yang, Erfu Wang, Yong Liu, and Qun Ding: "A Novel Multimodal Biometric System based on Iris and Face". International Journal of Digital Content Technology and its Applications (JDCTA), Volume6, Number2, February 2012.

[5] Mohamed K. Shahin, Ahmed M. Badawi, and Mohamed E. M. Rasmy. "Multimodal Biometric System Based on Near-Infra-Red Dorsal Hand Geometry and Fingerprints for Single and Whole Hands". World Academy of Science, Engineering and Technology, Vol: 5 2011-08-
23.

[6] D.J. Field. "Relation between the statistics of natural images and the response properties of cortical cells". J. Opt. Soc. Am. A, 4(12):2379_2394, 1987.

[7] Ojansivu, V., Heikkilä, J. "Blur Insensitive Texture Classification Using Local Phase Quantization". In Elmoataz, A., Lezoray, O., Nouboud, F., Mammass, D. (eds.) ICISP 2008 2008, LNCS, vol. 5099, pp. 236-243. Springer, Heidelberg (2008).

[8] Harbi AlMahafzah, Mohammad Imran, and H.S. Sheshadri "Multi-Algorithm Decision-Level Fusion Using Finger-Knuckle-Print Biometric". In T.h. Kim et al. (Eds): FGCN/DCA 2012, CCIS 350, pp. 302-311, 2012. () Springer-Verlag Berlin Heidelberg 2012.

[9] Zhifang Wang, Chao Liu, Taibin Shi and Qun Dingcanonical Face-Palm Identi_cation System on Feature Level Fusion based on CCA [Journal of Information Hiding and Multimedia Signal Processing].

[10] S.K.Bhardwaj, "An Algorithm for Feature Level Fusion in Multimodal Biometric System" International Journal of Advanced Research in Computer Engineering \& Technology (IJARCET) Volume 3 Issue 10, October 2014.

[11] Harbi AlMahafzah, H. S. Sheshadri and Mohammad Imran. "Multi-Algorithm Decision-Level Fusion Using Finger-Knuckle-Print Biometric" In V. Sridhar et al. (eds.), Emerging Research in Electronics, Computer Science and Technology, Lecture Notes in Electrical Engineering 248, DOI: 10.1007/978-81-322-1157-0_5, Springer India 2014.

[12] Nupriya Goyal, Dr. Rohit Bajaj: “An Improved Feature level Fusion in Multimodel Biometric System Using Iris and Ear". International Journal of Engineering Research and General Science Volume 3, Issue 3, May-June, 2015.

[13] AlMahafzah. H., Imran M., and Sheshadri, H.S. "Multibiometric: Feature Level Fusion Using FKP MultiInstance biometric". IJCSI International Journal of Computer Science. Issues Volume 9 Issue 4 No. 3, July 2012. 